\title{
锂硫电池循环过程中变形演化的直接观测
}

\author{
王宇晖 ${ }^{1,2}$, 靳 俊 ${ }^{2}$, 郭战胜 ${ }^{1}$, 温兆银 ${ }^{2}$
}

(1. 上海大学 上海市应用数学和力学研究所, 上海 $200072 ; 2$. 中国科学院 上海硅酸盐研究所, 中国科学院能量转换材 料重点实验室, 上海 200050)

摘 要: 实验设计了一种可实时原位观测锂硫电池电极变形及电化学反应过程的实验装置。实验中观测到充放电过 程中的硫电极的弯曲形变, 发现放电过程中硫正极形变增大, 曲率增大, 静置和充电过程中形变逐步还原, 曲率随 之减小。同时还发现随着电化学反应的进行, 由于放电产物多硫化物的溶解, 正极附近的电解液呈黄色且不断加 深。结合扫描电子显微镜(SEM)观察充放电前后的电极断面和表面的形貌，验证硫正极在充放电过程中出现明显的 形变, 从而导致电极曲率的变化。

关 键 词: 锂硫电池; 弯曲形变; 电极膨胀; 透明电池; 硫电极

中图分类号: O646 文献标识码: A

\section{Direct View for the Deformation Evolution of Sulfur Electrode during Li-S Battery Cycling}

\author{
WANG Yu-Hui ${ }^{1,2}$, JIN Jun ${ }^{2}$, GUO Zhan-Sheng ${ }^{1}$, WEN Zhao-Yin ${ }^{2}$
}

\begin{abstract}
(1. Shanghai Institute of Applied Mathematics and Mechanics, Shanghai University, Shanghai 200072, China; 2. CAS Key Laboratory of Materials for Energy Conversion, Shanghai Institute of Ceramics, Chinese Academy of Sciences, Shanghai 200050, China)
\end{abstract}

\begin{abstract}
An experimental set-up was developed which can observe electrode deformation and electrochemical reaction in-situ during the charge-discharge cycling of lithium-sulfur battery. The positive bending deformation during charge and discharge was monitored at real time. It was found that the positive curvature increased during discharge and reduced during open circuit. The curvature would recover the original state during charge period. As electrochemical reaction proceeds, the electrolyte near positive electrode gradually become yellow and deepened due to polysulfide dissolution of discharge products. Results of surface and section morphology observation on sulfur electrode before and after cycle using scanning electron microscope (SEM) were consistent with curvature changes during cycling and verified the volumetric expansion of sulfur electrode.
\end{abstract}

Key words: lithium-sulfur battery; bending deformation; electrode expansion; transparent battery; sulfur electrode

随着社会和经济的快速发展, 以及人们生活质 量的提高, 对能源的需求越来越大, 日常生活中便 携式电子设备以及电动汽车等对二次电池性能的要 求也越来越高 ${ }^{[1]}$, 因此迫切需要开发新型的高比能
电池。以单质硫与金属锂构成的二次电池体系的理 论比能量密度可达 $2600 \mathrm{Wh} / \mathrm{kg}$, 其中硫正极的理论 比容量是 $1675 \mathrm{mAh} / \mathrm{g}$ ，并具有低成本、环境友好等 特点, 成为近年来高能量密度二次电池领域中研究

收稿日期: 2016-05-17; 收到修改稿日期：2016-08-25

基金项目: 国家自然科学基金(51201177，51372262，11472165) National Natural Science Foundation of China (51201177, 51372262, 11472165)

作者简介: 王宇晖(1990-)，男，硕士研究生. E-mail: wangyuhui@student.sic.ac.cn

通讯作者: 郭战胜，教授.E-mail: davidzsguo@shu.edu.cn; 温兆银, 研究员.E-mail: zywen@mail.sic.ac.cn 
的热点 ${ }^{[2-5]}$ 。

虽然锂硫电池具有很高的能量密度, 但仍有一 系列技术难题函待解决。硫在锂化过程中生成的 $\mathrm{Li}_{2} \mathrm{~S}_{x}(4 \leqslant x \leqslant 8)$ 会溶解在电解液中, 并扩散到负极与 金属锂直接反应，生成不溶的 $\mathrm{Li}_{2} \mathrm{~S}_{y}(y \leqslant 2)$, 这样一 方面造成锂负极的腐蚀和活性物质的损耗, 另一方 面在电池充电过程中 $\mathrm{Li}_{2} \mathrm{~S}_{y}$ 又会嵌锂生成可溶解的 $\mathrm{Li}_{2} \mathrm{~S}_{x}$, 进而在充电过程中 $\mathrm{Li}_{2} \mathrm{~S}_{x}$ 和 $\mathrm{Li}_{2} \mathrm{~S}_{y}$ 相互转化, 即所谓的 “穿梭效应”, 成为造成电池容量衰减和 库伦效率降低的主要原因之一[3, 6-8]。与硅、锡负极类 似, 硫在锂化过程中存在严重的体积膨胀, 使得硫正 极在循环过程中出现反复的体积膨胀收缩现象 ${ }^{[3,9]}$, 导致电极结构损坏或电极与集流体分离, 进而影响 电池的循环性能。He 等 ${ }^{[9]}$ 通过实验观察发现硫复合 电极在放电时厚度增加了约 $22 \%$, 充电时则收缩。 但到目前为止, 研究人员还未能在线观察锂硫电池 充放电过程中实际发生的体积变化及其相关反应。

针对锂硫电池中的穿梭效应和硫正极的体积膨 胀问题, 本工作设计了一种特殊结构的透明电池, 使正极在固定后成悬臂梁结构, 在充放电过程中可 在线观测正极的形变情况。通过建立坐标系, 根据正 极所处位置的坐标计算在不同时刻下的平均曲率。 最终得到正极的曲率随时间变化的规律, 并通过扫 描电子显微镜(SEM) 观察电极形貌及厚度变化。

\section{1 实验方法}

\section{1 实验材料}

硫电极的组成与制备采用本实验室优化的体系 与方法 ${ }^{[10-14]}$ 。制备正极浆料时, 将硫碳复合材料(硫 含量 75\%)0.8 g、导电炭黑 $0.05 \mathrm{~g}$ 、碳纳米管 $0.05 \mathrm{~g}$ 、 LA132 水性粘结剂(5\%溶液) $2 \mathrm{~g}$ 和 $1.1 \mathrm{~mL}$ 去离子水 混合, 摚拌 $12 \mathrm{~h}$ 。将得到的浆料均匀涂覆在 $20 \mu \mathrm{m}$ 厚 的铝箔上, 涂布厚度为 $90 \mu \mathrm{m}, 60^{\circ} \mathrm{C}$ 真空干燥 $12 \mathrm{~h}$ 。干 燥后将正极裁剪成 $3 \mathrm{~mm} \times 30 \mathrm{~mm}$ 的矩形, 并预留一 定长度的铝箔用来焊接极耳。负极为金属锂, 隔膜 为涂覆氧化铝的陶瓷隔膜。电解液为 $1 \mathrm{~mol} / \mathrm{L}$ 的双 三氟甲基磺酰亚胺锂(LiTFSI)/乙二醇二甲醚(DME)+ 1,3 -二氧戊环 $(\mathrm{DOL})($ 体积比为 $1: 1)$ 。

\section{2 电池组装}

在充满氩气的手套箱中组装电池, 水和氧气含 量均小于 $1 \times 10^{-6}$ 。如图 1(a)所示, 将材料按照正极、 隔膜、负极的顺序叠放在一起组装成电池, 并用石 英片固定, 使负极一侧与石英片接触, 正极一侧不 加限制, 成悬臂梁结构, 使其可以在充放电过程中

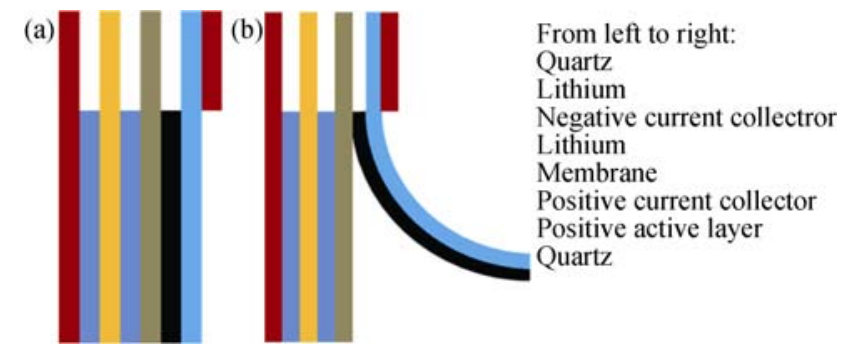

图 1 电池组装示意图

Fig. 1 Schematic of battery assembly (a) before and (b) after transformation

自由变形, 变形后如图 1(b)所示。组装的电池静置 $12 \mathrm{~h}$ 后进行测试。使用 BK-6808 电池测试系统进行 充放电试验, 电池以 $0.2 C$ 倍率循环, 截止电压范围 为 $1.0 \sim 2.6 \mathrm{~V}$ 。

\section{3 形变测量}

使用 $\mathrm{CCD}$ 实时监测电池充放电过程中电极的 形变及电化学反应后电解液的变化。每隔 $30 \mathrm{~s}$ 照相 记录正极的形变状态。建立如图 2 所示的坐标系, 其中矩形代表负极与隔膜, 黑色曲线为正极, 在曲 线上取间隔均匀的 180 个点的坐标值, 利用三点确 认一个圆即得到一个曲率值, 将曲率值取平均值得 到相应时刻在此坐标系下正极的曲率。最后再用此 坐标系下求的曲率乘以放大倍数, 即实际物体的尺 寸在照片中的放大倍数, 可以得到正极在此时刻的 平均曲率。

\section{4 微观形貌表征}

使用与观测形变相同的方式组装电池, 通过扫 描电子显微镜(SEM, 型号为 Hitachi S-3400N)分析 正极在循环前后表面与断面的显微结构。为了准确 反映充放电过程中电极的微结构变化, 在放电前对 制成的电极进行切割。断面的一侧用于放电前分析, 另一侧则用于放电后的微结构分析。

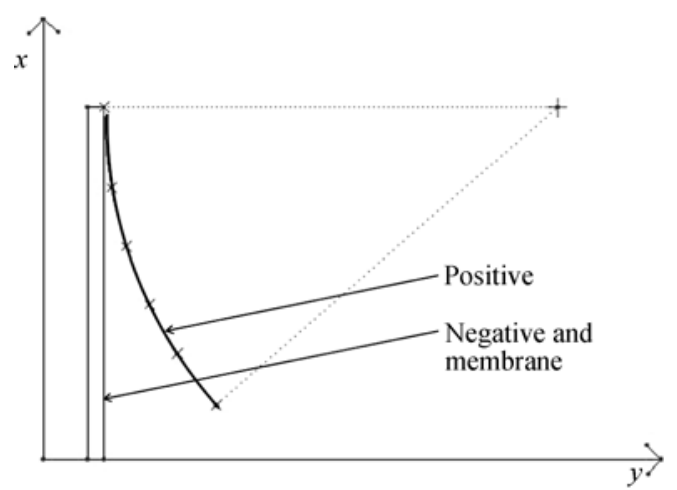

图 2 形变监测及曲率计算示意图

Fig. 2 Schematic of deformation monitoring and curvature calculation 


\section{2 实验结果}

\section{1 充放电过程的电化学反应以及电解液变 化观测}

典型的锂硫电池包括金属锂负极, 有机电解液, 含硫复合正极。放电过程中, 金属锂负极进行锂的电 化学溶解过程并释放电子。锂离子通过电解液输运到 正极, 电子通过外部电路运动到正极, 形成放电电 流。硫在正极与锂离子和电子生成锂硫化物。放电反 应的化学方程式如下所示, 充电反应为其逆反应。

阳极反应: $2 \mathrm{Li} \rightarrow 2 \mathrm{Li}^{+}+2 \mathrm{e}^{-}$

阴极反应: $\mathrm{S}+2 \mathrm{Li}^{+}+2 \mathrm{e}^{-} \rightarrow \mathrm{Li}_{2} \mathrm{~S}$

$$
\text { 总反应： } 2 \mathrm{Li}+\mathrm{S} \rightarrow \mathrm{Li}_{2} \mathrm{~S}
$$

图 3 是锂硫电池前两次充放电曲线。通常锂硫 电池 ${ }^{[15-18]}$ 放电曲线由两个平台组成, 第一个放电平 台的电压范围在 2.4 2.1 V, 元素硫被还原成可溶性 的多硫化锂, 并且多硫化锂会被进一步还原。在高 电压平台每个硫原子接受 0.5 个电子可以将起始正 极材料环状 $\mathrm{S}_{8}$ 分子还原成 $\mathrm{Li}_{2} \mathrm{~S}_{4}$, 对应这个过程的 电化学反应为:

$$
\mathrm{S}_{8}^{0}+4 \mathrm{e}^{-}=2 \mathrm{~S}_{4}^{2-}
$$

每个硫原子接受 0.5 个电子也可解释为高放电 平台的硫由 $\mathrm{S}_{8}$ 和 $x>4$ 的聚硫离子 $\mathrm{S}_{x}^{2-}$ 组成。此时, 在 正极附近的电解液中开始出现黄色物质, 图 4(b)中 照片 2。黄色物质的主要成分为多硫化锂 ${ }^{[19-22]}$ 。第 二个放电平台的电压范围在 $2.1 \sim 1.5 \mathrm{~V}$, 可溶性聚硫 锂子被还原成硫化锂层覆盖在碳骨架上。低放电平台 为硫原子接受另一个电子, 进行 $\mathrm{Li}_{2} \mathrm{~S}_{4}$ 的还原, 生成 $\mathrm{Li}_{2} \mathrm{~S}$ 和 $\mathrm{Li}_{2} \mathrm{~S}_{2}$ 的混合物。低放电平台的反应方程为:

$$
\mathrm{S}_{4}^{2-}+4 \mathrm{e}^{-}=2 \mathrm{~S}^{2-}+\mathrm{S}_{2}^{2-}
$$

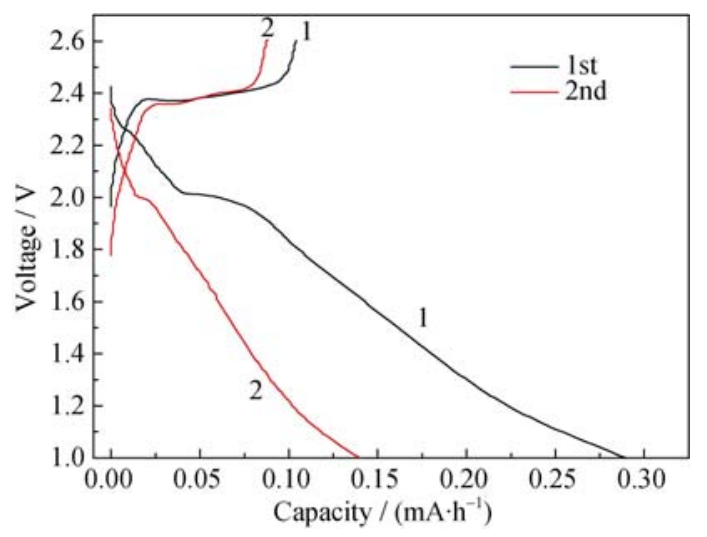

图 3 锂硫电池第一次与第二次循环充放电曲线

Fig. 3 First and second charge-discharge curves of Li-S battery
此时，正极中依然会有黄色物质不断生成，并 溶解在电解液中。第二个平台结束后, 放电电压持 续下降, 黄色物质逐渐溶解在电解液中, 如图 4(b) 照片 3 5, 反映了电解液中多硫化锂的变化过程。放 电结束后, 电解液透明, 溶解的多硫化锂均匀溶解 到电解液中。充电过程中, 又会有黄色物质溶解在 电解液中, 主要是硫化锂反应生成的多硫化锂。这 种透明电池的电解液相比于纽扣电池的含量要多, 通常纽扣电池中 $1.2 \mathrm{mg}$ 硫对应 $80 \mu \mathrm{L}$ 电解液, 而在 透明电池中 $2.3 \mathrm{mg}$ 硫对应 $60 \mathrm{~mL}$ 的电解液, 大量的 多硫化物溶解在电解液中, 对电池的充放电性能造 成影响。该现象是图 3 中两次循环之间电池容量下 降的主要原因, 即中间产物多硫化锂溶解在电解液 中, 降低了电极中活性物质的质量, 致使电池容量 迅速下降。此外, 第一次放电过程中伴随的激活活 性颗粒和其他副反应也是造成第二次放电容量明显 小于第一次放电容量的原因之一。

\section{2 变形演化}

电池在充放电过程中，集流体铝簿不会由于电 子传输而变形, 只有正极活性层由于锂离子的嵌入 与脱嵌导致活性层膨胀和收缩，进而使得紧密贴合 的电极活性层由于反应过程中的应力与集流体一起 发生弯曲变形。

电池在首次循环充放电过程中, 第一步以 $0.2 \mathrm{C}$ 放电, 截止电压为 $1.0 \mathrm{~V}$ 。放电过程中, 正极由初始 位置逐渐弯曲, 当电压降到 $1.0 \mathrm{~V}$ 时达到最大形变, 即弯曲程度最大如图 4(b)中照片 1 5。在放电过程 中, 硫与锂反应生成多硫化锂并最后生成硫化锂的 整个过程中体积逐渐膨胀, 使得活性层发生膨胀现 象, 进而使得活性层与铝䇴发生弯曲变形。第二步 为 $10 \mathrm{~min}$ 的静置过程，电极的形变有一定程度的恢 复, 同时伴随有电压增加。在静置过程中, 活性层中 的各离子浓度梯度降低, 电解液和活性层中发生的 反应使正极中的硫化锂向多硫化锂转化使锂含量减 少, 或结构应力松驰等原因都会造成形变恢复。第 三步以 $0.2 \mathrm{C}$ 充电, 截止电压为 $2.6 \mathrm{~V}$ 。充电过程中, 正极的形变继续恢复, 如图 4(b)中照片 6 和 7。充电 过程与放电过程电极的形变正好相反, 硫化锂生成 多硫化锂并最后转化为硫, 由于生成的硫密度大于 硫化锂, 电极活性层体积收缩, 使得整个电极形变 得到恢复。

通过对拍摄的每张照片进行取点分析, 计算得 到正极的曲率，可以得到正极在循环过程中的曲率 变化, 如图 4(a)所示。在放电阶段, 与放电曲线中两 个放电平台相对应的曲率变化较为平缓。当电压下 


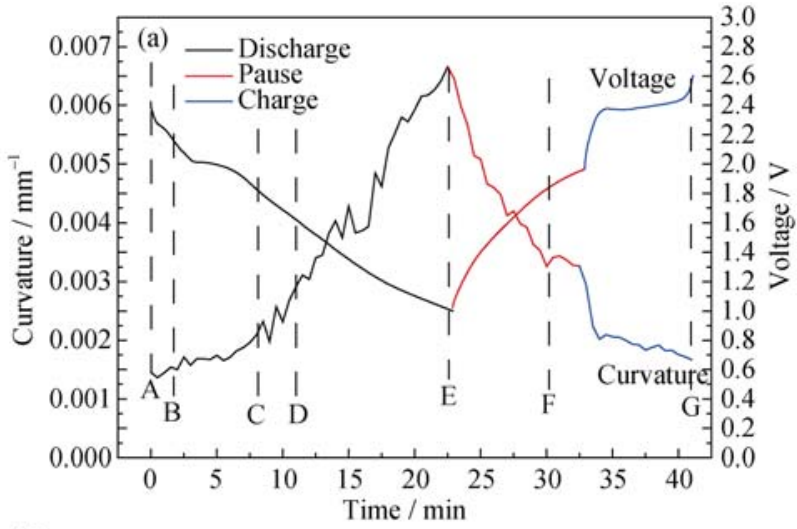

(b)

\begin{tabular}{|c|c|c|c|c|c|c|}
\hline $\mathrm{A}$ & $\mathrm{B}$ & $\mathrm{C}$ & $\mathrm{D}$ & $\mathrm{E}$ & $\mathrm{F}$ & $\mathrm{G}$ \\
\hline $0 \mathrm{~min}$ & $2 \mathrm{~min}$ & $9 \mathrm{~min}$ & $13 \mathrm{~min}$ & $23 \mathrm{~min}$ & $33 \mathrm{~min}$ & $41 \mathrm{~min}$ \\
\hline & & & & & & \\
& & & & & & \\
& & & & & & \\
& & & & & & \\
\hline
\end{tabular}

图 4 电压、电化学反应及电极形变与充放电时间的关系曲线 Fig. 4 Relation curves between voltage, electrochemical reaction, electrode deformation, and cycle time

(a) Changes in voltage and positive deformation over time under complete charge and discharge cycle; (b) Portion screenshot of electrochemical reaction and electrode deformation during cycle
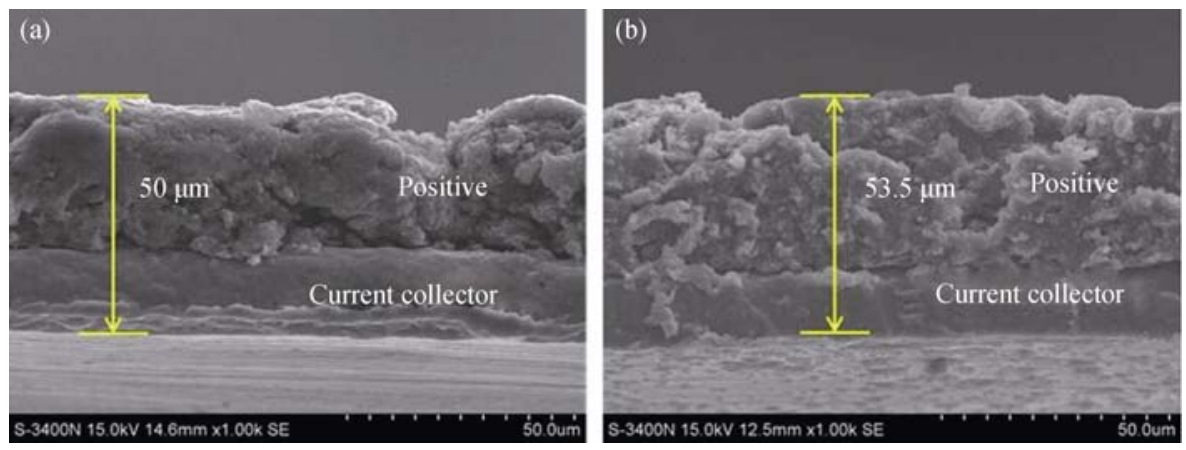

图 5 电极横断面厚度变化 SEM 照片

Fig. 5 Cross section showing thick changing of S electrode (a) Before 1st charge-diacharge cycle; (b) After 1st charge-diacharge cycle
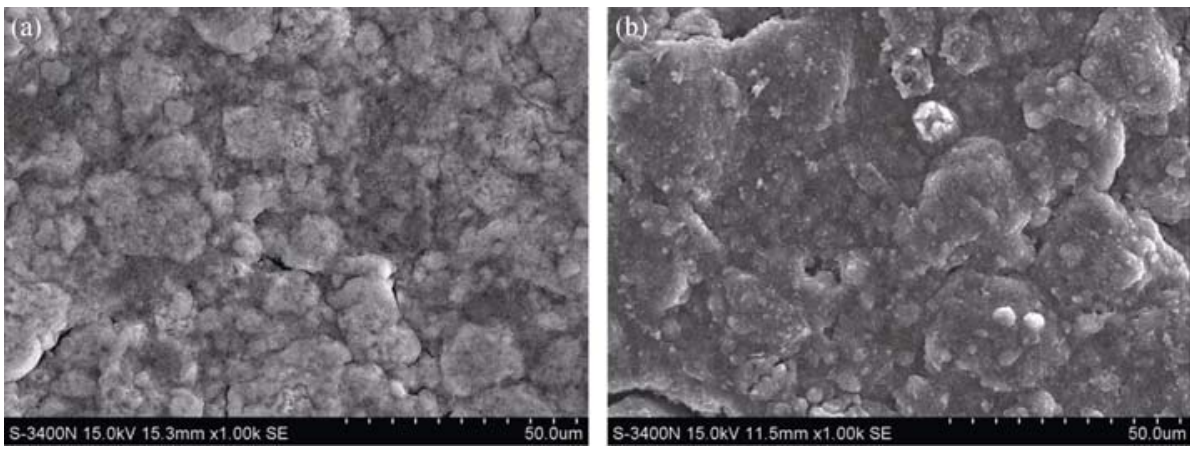

图 6 电极表面形貌的 SEM 照片

Fig. 6 Surface morphologies of S electrode (a) Before 1st charge-diacharge cycle; (b) After 1st charge-diacharge cycle 
形貌的变化。对比循环前后的断面与表面发现, 循 环后与循环前相比不是很平整，可能的原因如下: 一方面 $\mathrm{S}$ 生成的 $\mathrm{Li}_{2} \mathrm{~S}_{2}$ 与 $\mathrm{Li}_{2} \mathrm{~S}$ 存在体积膨胀且在充 电过程中并未完全转化成 $\mathrm{S}$; 另一方面电解液中的 溶剂, 锂盐自身的分解沉积在表面 ${ }^{[23-25]}$ 。

\section{3 结论}

实验设计了能够实时观测锂硫电池充放电循环 过程中硫正极的变形演化装置。结果表明, 硫正极 在放电过程中电极曲率逐渐增大, 静置和充电过程 中曲率逐渐减小至原来的状态, 且电池电压变化越 快, 曲率变化也越快。结合 SEM 观测硫电极充放电 前后的横截面厚度变化, 验证了放电反应导致的体 积膨胀。此外, 设计的实验装置还直接观测到了锂 硫电池的放电中间产物多硫化锂在电解液中的溶解 现象。

\section{参考文献:}

[1] LIN CHEN, LEON L SHAW. Recent advances in lithium-sulfur batteries. Journal of Power Sources, 2014, 267: 770-783.

[2] ROSENMAN ARIEL, ELAZARI RAN, SALITRA GREGORY, et al. The effect of interactions and reduction products of $\mathrm{LiNO}_{3}$, the anti-shuttle agent, in Li-S battery systems. Journal of The Electrochemical Society, 2015, 162(3): A470-A473.

[3] YANG YUAN, ZHENG GUANG-YUAN, CUI YI. Nanostructured sulfur cathodes. Chemical Society Reviews, 2013, 42(7): 3018-3032.

[4] MANTHIRAM ARUMUGAM, FU YONG-ZHU, CHUNG SHENG-HENG, et al. Rechargeable lithium-sulfur batteries. Chemical Reviews, 2014, 114(23): 11751-11787.

[5] XU GUI-YIN, DING BING, PAN JIN, et al. High performance lithium-sulfur batteries: advances and challenges. Journal of Materials Chemistry A, 2014, 2(32): 12662-12676.

[6] YIN YA-XIA, XIN SEN, GUO YU-GUO, et al. Lithium-sulfur batteries: electrochemistry, materials, and prospects. Angewandte Chemie International Edition, 2013, 52(50): 13186-13200.

[7] LI WEI-YANG, ZHANG QIAN-FAN, ZHENG GUANG-YUAN, et al. Understanding the role of different conductive polymers in improving the nanostructured sulfur cathode performance. Nano Letters, 2013, 13(11): 5534-5540.

[8] BRUCE PETER G, FREUNBERGER STEFAN A, HARDWICK LAURENCE $\mathrm{J}$, et al. $\mathrm{LiO}_{2}$ and $\mathrm{Li}-\mathrm{S}$ batteries with high energy storage. Nat-Mater., 2012, 11(1): 19-29.

[9] HE XIANG-MING, REN JIAN-GUO, WANG LI, et al. Expansion and shrinkage of the sulfur composite electrode in rechargeable lithium batteries. Journal of Power Sources, 2009, 190: 154-156.

[10] JIN JUN, WEN ZHAO-YIN, MA GUO-QIANG, et al. Mesopor- ous carbon/sulfur composite with polyaniline coating for lithium sulfur batteries. Solid State Ionics, 2014, 262: 170-173.

[11] MA GUO-QIANG., WEN ZHAO-YIN, JIN JUN, et al. Enhanced performance of lithium sulfur battery with polypyrrole warped mesoporous carbon/sulfur composite. Journal of Power Sources, 2014, 254: 353-359.

[12] MA GUO-QIANG, WEN ZHAO-YIN, JIN JUN, et al. Enhancement of long stability of Li-S battery by thin wall hollow spherical structured polypyrrole based sulfur cathode. RSC Advances, 2014, 4(41): 21612-21618.

[13] WANG QING-SONG, JIN JUN, WU XIANG-WEI, et al. A shuttle effect free lithium sulfur battery based on a hybrid electrolyte. Physical Chemistry Chemical Physics, 2014, 16(39): 2122521229.

[14] JIN JUN, WEN ZHAO-YIN, MA GUO-QIANG, et al. Flexible self-supporting graphene-sulfur paper for lithium sulfur batteries. RSC Advances, 2013, 3(8): 2558-2560.

[15] CHEON SANG-EUN, KO KI-SEOK, CHO JI-HOON, et al. Rechargeable lithium sulfur battery: I. structural change of sulfur cathode during discharge and charge. Journal of The Electrochemical Society, 2003, 150(6): A796-A799.

[16] CHEON SANG-EUN, KO KI-SEOK, CHO JI-HOON, et al. Rechargeable lithium sulfur battery: II. Rate capability and cycle characteristics. Journal of The Electrochemical Society, 2003, 150(6): A $800-A 805$.

[17] MIKHAYLIK YURIY V, AKRIDGE JAMES R. Polysulfide shuttle study in the Li/S battery system. Journal of The Electrochemical Society, 2004, 151(11): A1969-A1976.

[18] MANTHIRAM ARUMUGAM, FU YONGZHU, SU YU-SHENG. Challenges and prospects of lithium-sulfur batteries. Accounts of Chemical Research, 2013, 46(5): 1125-1134.

[19] LI YA-JUAN, ZHAN HUI, LIU SU-QIN, et al. Electrochemical properties of the soluble reduction products in rechargeable $\mathrm{Li} / \mathrm{S}$ battery. Journal of Power Sources, 2010, 195(9): 2945-2949.

[20] TOBISHIMA SHIN-ICHI, YAMAMOTO HIDEO, MATSUDA MINORU. Study on the reduction species of sulfur by alkali metals in nonaqueous solvents. Electrochimica Acta, 1997, 42(6): 1019-1029.

[21] DIAO YAN, XIE KAI, XIONG SHI-ZHAO, et al. Analysis of polysulfide dissolved in electrolyte in discharge-charge process of Li-S battery. Journal of the Electrochemical Society, 2012, 159(4): A421-A425.

[22] SUO LIU-MIN, HU YONG-SHENG, LI HONG, et al. A new class of solvent-in-salt electrolyte for high-energy rechargeable metallic lithium batteries. Nat. Commun., 2013, 4: 1481.

[23] JI XIU-LEI, LINDA F. NAZAR. Advances in Li-S batteries. Journal of Materials Chemistry, 2010, 20(44): 9821-9826.

[24] DORON AURBACH, ELAD POLLAK, RAN ELAZARI, et al. J. On the surface chemical aspects of very high energy density, rechargeable Li-Sulfur batteries. Journal of The Electrochemical Society, 2009, 156(8): A694-A702.

[25] JI XIU-LEI, KYU TAE LEE, LINDA F NAZAR. A highly ordered nanostructured carbon-sulphur cathode for lithium-sulphur batteries. Nat. Mater., 2009, 8(6): 500-506. 